\title{
Development of an Intelligent Hypertext Manual for the Space Shuttle Hazardous Gas Detection System
}

\author{
SEMI-ANNUAL REPORT
}

Covering the period July 1, 1992 through December 31, 1992

Principal Investigator:

Dr. C.F. Lo, University of Tennessee Space Institute

Under NASA Marshall Space Flight Center NAG 8-835

Marshall Space Flight Center, AL 35812

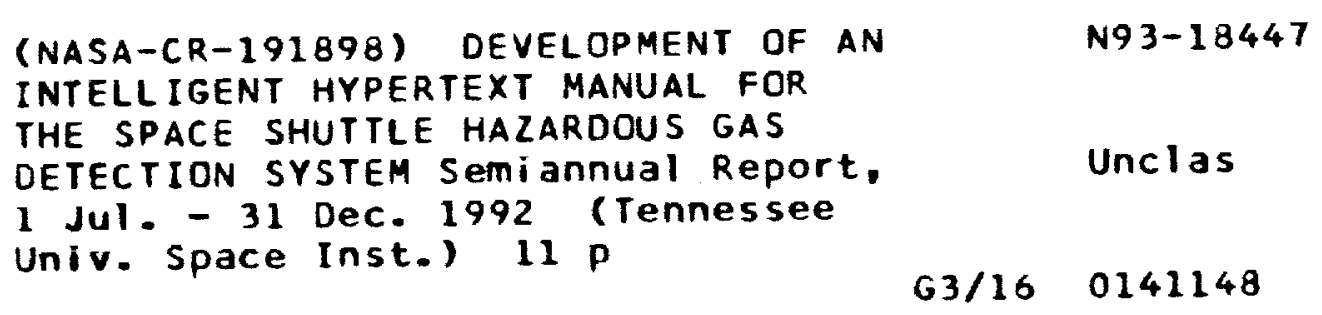

\section{INSTITUTION:}

Center for Space Transportation and Applied Research

UTSI Research Park

Tullahoma, TN 37388-8897

Phone 615-454-9294 


\title{
Development of an Intelligent Hypertext \\ Manual for the Space Shuttle Hazardous Gas Detection System
}

NASA NAG 8-835

Semi-Annual Progress Report

July 11992 through December 31, 1992

\section{Investigators:}

Dr. Ching F. Lo

Principal Investigator, CSTAR/UTSI

Dr. George Z. Shi

Mr. Carl Bangasser

Ms. Connie Fensky

Mr. Hugh Campbell

\author{
Research Engineer, CSTAR/UTSI \\ Graduate Research Assistant, CSTARUTSI \\ Graduate Research Assistant, CSTAR/UTSI \\ Technical Monitor, NASA MSFC
}

\section{Technical Objective:}

The objective is to create a computerized service manual in the form of a combination of Hypertext and expert system which stores experts' knowledge and experience for hazardous gas detection and analysis. The resulting Intelligent Hypertext Manual (IHM) will:

1) locate applicable documentation and previous fault histories quickly.

2) recognize and identify possible hazardous gas leak problems during shuttle launch operations.

3) assist in training personnel.

4) easily accommodate updates and further development.

\section{Rockwell Visit}

On August 11, 1992, a CSTAR team visited the Rockwell operation in Huntsville to meet with Mr. Tom Day and other Rockwell personnel to present our progress and discuss future plans. At this meeting, a working version of the IHM was demonstrated and ideas for future development were discussed. CSTAR received several Quick-Look Reviews for recent shuttle missions during the visit.

\section{Progress}

The specific areas in which progress was made are described below. A paper, which was presented at the CSTAR Third Annual Technical Symposium, November 11-12, 1992, is enclosed as an Appendix to describe the progress in detail.

\subsection{IHM's User Interface Update}

During this report time period, the IHM's user-interface has been re-built using ToolBook. ToolBook has been chosen to succeed Guide 3 because:

1) ToolBook has more functions and is more flexible than Guide 3 ,

2) a ToolBook interface is much easier to use and understand,

3) ToolBook has much better programming capabilities. 
Guide 3 will still be used for long text documents such as technical papers, but it will be called from the ToolBook interface.

\subsection{IHM System Accessories Addition}

The IHM's system accessories such as Help, Index, Glossary, and Map are fully functional now. These accessories are designed to increase the IHM's efficiency and make it more user-friendly. Because of the nature of the IHM, these accessories are in hypertext format and the user may access these accessories from anywhere within the IHM.

The context-sensitive Help provides help depending on where the user is in the system. Users can easily get the specific help they need rather than search through long help-menu screens. The Index is an alphabetical list of topics contained within the IHM. The hypertext nature of the Index allows the user to directly access the topic he/she wishes to see by clicking on its name listed in the Index. The Map is another navigational aid; it shows a graphical representation of the system and allows the user to travel to different locations within the system by clicking on the appropriate icons.

\subsection{MPS Document Figure Addition}

Additional schematics and figures from the Rockwell International MPS/Flight Measurement Location Document, which were not supplied electronically, have been scanned into the computer. They are now being linked into the hypertext MPS/MLD.

\subsection{Working Copy Ready for Testing}

A working copy of the IHM has been installed on a Dell $386 \mathrm{PC}$ and is ready to be delivered to NASA Marshall for beta-testing. The feedback from NASA engineers will be incorporated into system improvements.

\section{Future Plans}

Future effort in the development of the IHM during the remaining project time period will be:

1. Incorporate the feedback from NASA engineers into the IHM.

2. Build additional Quick-Look Reviews according to Rockwell or NASA requests.

3. Build utility tools for system maintenance and update, such as LCC documents updating and Quick-Look Review building.

4. Study the requirements for including plotting capabilities for near-real-time gas measurement data into the IHM. 


\title{
An Integrated Knowledge System for the Space Shuttle Hazardous Gas Detection System
}

\author{
Nov. 11-12, 1992 \\ Ching F. Lo', George Z. Shi², \\ Carl Bangasser ${ }^{3}$, and Connie Fensky ${ }^{3}$ \\ UT-Calspan Center for Space Transportation and Applied Research \\ Tullahoma, TN 37388-8897
}

\begin{abstract}
A computer-based Integrated Knowledge System (IKS), the Intelligent Hypertext Manual (IHM), is being developed for the Space Shuttle Hazardous Gas Detection System (HGDS) at the Huntsville Operations Support Center (HOSC). The IHM stores all HGDS related knowledge and presents them in an interactive and intuitive manner. The IHM's purpose is to provide HGDS personnel with the capabilities of: enhancing the interpretation of real time data; recognizing and identifying possible faults in the Space Shuttle sub-systems related to hazardous gas detections; locating applicable documentation related to procedures, constraints, and previous fault histories; and assisting in the training of personnel.
\end{abstract}

\section{Introduction}

Space Shuttle hazardous gas leakage monitoring is a complicated process which involves a large collection of hardware, documents, and experts' knowledge. There is a pressing need to improve the Hazardous Gas Detection System's (HGDS) data interpretation, fault recognition, and corrective action assistance. A computer-based integrated knowledge system, the Intelligent Hypertext Manual (IHM), may provide just the right tool to accomplish this task.

The IHM is a hypermedia system designed to store information related to the HGDS and to present it in an interactive and intuitive manner. The IHM has a friendly user-interface, a set of system accessories, and a knowledge base which consists of many information and knowledge elements. The fundamental principle of the IHM is the efficient organization, representation, and utilization of its knowledge. The system architecture of the IHM is shown in Figure 1. The basic design idea is to construct the IHM using commercial software shells, which are well developed and available for all potential users, so the developer can concentrate on developing the knowledge-elements and integrating them into the IHM. The IHM is built with an open architecture so that the new knowledge-elements can be easily integrated into the system. This greatly simplifies the task of maintaining and updating the IHM.

The IHM will also assist users in the decision-making process; it provides "quick and easy" access to appropriate information for fault detection with action options. It may also supply a

${ }^{1}$ Principal Investigator, CSTAR, Professor of AE/ME, UTSI.

2 Research Engineer, CSTAR.

${ }^{3}$ Graduate Research Assistants, CSTARUTSI 
large volume of accessible material in specific areas of space shuttle hardware for assistance in training personnel.

In short, the IHM has the potential to increase productivity and the quality of service for fault detection from the HGDS. It eliminates most human errors and reduces the required resources including manpower and material cost to achieve the same specified goal.

\section{Hypermedia Technology}

The basic technology which makes the information presentation of the IHM possible is hypermedia. Hypermedia incorporates information in an enriched environment that complements text with high quality pictures, full motion video, and sound. More importantly, hypermedia allows the reader to browse through linked, cross-referenced, annotated, and layered information quickly in a non-sequential manner. Figure 2 is an illustration of hypermedia. Information within a hypermedia document contains facts (such as notes, papers, books, diagrams, photos, full motion video and sound, and other software packages) and the relationships between those facts. By following the relationships between facts, the reader has the ability to find and display information in an intuitive and interactive manner. The hypertext, which is used for the IHM, is one branch of hypermedia that deals mainly with text and graphics(Ref. 1 - 3).

Hypermedia is a very flexible tool that can be applied to a wide range of applications. Essentially any information that can be presented electronically can benefit from hypermedia. Theoretically, hypermedia systems have no size limitation; therefore, they can be expanded to link every piece of electronic information available. A few areas in which hypermedia is often used are technical reference materials, maintenance manuals, and policies and procedures manuals.

\section{Implementation}

The IHM currently operates on the IBM PC platform. The Microsoft Windows 3.1 (Ref. 4) graphical environment was selected to be the working environment and the user-interface was built using ToolBook (Ref. 5) and Guide 3 (Ref. 6). Other application software included are an expert system shell and a plotting program.

\section{IHM's User-Interface}

The design of the user-interface is one of the most important aspects in developing the IHM because the user relies on it for information retrieval. The IHM's user-interface is constructed to provide easy access to all knowledge-elements such as hypertext documents, databases, expert systems, etc., and to allow the user to navigate through the system. Therefore, the interface must be simple to use and easy to understand.

The layout and operation of the IHM's user-interface is consistent throughout the entire system to promote user familiarity. The information in the IHM is formatted in distinct, screen-size pieces (or "pages") so that it can be instantly available and easily assimilated by the user. The main elements of the user-interface are buttons or other types of instant command objects which 
carry out pre-defined actions. The IHM's user-interface has two types of these objects, navigational and special function buttons. The navigational buttons provide quick and easy access to any piece of information within the IHM. These type of buttons are consistent in their location and function throughout the IHM. The special-function buttons are specific to a certain page or page-type. They carry out the operational needs of the page in which they are contained.

During the second year of this research program, the IHM's user-interface has been re-built using ToolBook. ToolBook has been chosen to succeed Guide 3 because:

1) ToolBook has more functions and is more flexible than Guide 3.

2) a ToolBook interface not only appears more professional, but is easier to use and understand.

3) the scripting language used by ToolBook is easier to program than that used by Guide 3.

Guide 3 may still be used to present long text documents such as technical papers, but it will be called from the ToolBook interface.

\section{Knowledge-Elements and Organization}

Related information or knowledge are grouped into knowledge-elements or objects in the objectoriented environment of the IHM, as illustrated in the second layer of Figure 1. The knowledgeelements may be built using different software shells, each according to their own usage and nature. Each knowledge-element is linked to other knowledge-elements both by the userinterface and by the links within the elements. These links are made during system construction and are implicit to the users.

The knowledge-elements of the IHM contain all the critical information related to the HGDS, including information from applicable manuals, procedures, criteria, and data. The information included in the IHM are selected based on the access requirement priority in the hazardous gas leakage monitoring process and their availability.

\subsection{Hypertext Documents}

The hypertext documents are constructed from existing computerized and/or printed documents. The hypertext documents were built using ToolBook, an application construction software, and Guide 3, a hypertext software package. Both are designed for use in the Microsoft Windows graphical environment. Figure 3 shows the overall structure of the IHM knowledge base. The specific documents that have been included in the IHM are the following:

\section{Launch Commit Criteria (LCC) documents:}

The LCC documents are the most important documents used during the launch monitoring. The sections of the LCC/HAZ and LCC/GSE have been implemented into hypertext form and arranged in such a way that the user can easily access any single document either by clicking on its name or by the situation it covers during the launch, e.g. launch period, gas type, and sensor location. Figure 4 shows the hypertext form of a LCC document. 


\section{MPS/Flight Measurement Location Document:}

The MPS/Flight Measurement Location Document had been built into hypertext document format. The original Rockwell, Inc., documents were converted to Windows-readable graphicimage files. Hypertext buttons facilitate movement either sequentially through the document, or directly to a pertinent section via menus. Figure 5 is a screen display of a MPS/MLD figure. An automatic feature is included to easily update the document by incorporating new graphics images when desired.

\section{Operations and Maintenance Requirements and Specification Document:}

These documents include information pertaining to events occurring before External Tank loading, during the T-6 to T-4 hr time frame, and during the T-10 to T-1 minute time frame.

\section{Quick-Look Reviews:}

The Quick-Look Reviews for several launches have been incorporated into the IHM. These hypertext documents include the written prefaces and tables as well as the graphs that make up the bulk of these reports. The hypertext Quick-Look Manual allows users to navigate quickly to the portion they want to review. Figure 6 shows an example of a hypertext Quick-Look preface.

\subsection{Expert System for Data Retrieval}

The information of hazardous gas leakage measurements for all shuttles' loading operations has been built into a dBASE file for easy access. For future updates of this information, only this database need be changed. The ToolBook interface is designed to retrieve the data intelligently from the database file. The user can quickly retrieve the data sorted in several ways, such as by the launch vehicle, date, launch I.D., or by the data limit. The retrieved data is written into an ASCII report file and can be read, printed, or plotted as the user chooses.

\section{IHM System Accessories}

System accessories, such as Help, Index, Glossary, and Map are designed to increase the IHM's efficiency and make it more user-friendly. Because of the nature of the IHM, these accessories are in hypertext format and the user may access these accessories from anywhere within the IHM.

The Index is an alphabetical list of topics contained within the IHM. The hypertext nature of the Index allows the user to navigate directly to the topic he/she wishes to see by clicking on the topic. A Map is another such navigational aid, except that it shows a graphical representation of the system and allows travel to different locations within the system. The context-sensitive Help provides assistance depending on where the user is in the system. Users can easily get the specific help they need rather than search through long help-menu screens.

\section{IHM Plotting Upgrade}

The IHM's plotting ability has been upgraded with Origin (Ref. 7), a new Windows-based scientific and technical graphics software package by MicroCal, Inc. The user is able to: zoom 
to portions of the graph, view the numerical data, print portions of the plot, and manipulate the data with curve-fitting, statistics, arithmetic, etc. The user can also pre-program the tasks they desire using Origin's macro capability.

This upgrade was performed for a number of reasons. The transition from the IHM into Origin is much smoother because it stays in Windows and does not need to shell to DOS. Origin's user interface is much more "friendly" and supports the use of a mouse. Origin also allows for preprogramming, which reduces the amount of input and learning required for the user to plot data in the IHM.

The IHM has been set up to run Origin directly when plotting the data sorted by the expert system. Upon choosing the plot command in the IHM, Origin is launched and the data is automatically loaded. A plot is then automatically produced and scaled for that data. Figure 7 is a screen-print of a plot of sorted data from an expert system session.

The IHM also allows user to plot off-line, time dependent gas concentration data for a single launch. This gives the user a quick way of reviewing this form of the gas concentration data.

\section{Hypermedia Incorporation}

The possibility of incorporating full motion video and sound into the IHM has also been studied. A VideoLogic DVA-4000 video board and MIC System software (Ref. 8) have been installed on our IBM PC-386 computer. A laser-disc player is used to retrieve video images from pre-made laser discs. The command-buttons, which launch the player and put the related video images on the computer screen, are placed within the text documents so the user can view these images while reading the documents by simply clicking the buttons. This would give the IHM the capability of demonstrating a particular task to the user visually, rather than attempting to relate the task strictly with words. A photograph of a sample screen display is shown in Figure. 8. We believe that the resulting hypermedia document could be extremely useful and much more efficient.

\section{Concluding Remarks and Future Work}

Integration of computer and Artificial Intelligence technologies, in the form of the Integrated Knowledge System, holds much promise in improving the efficiency of personnel and increasing the productivity in aerospace applications. A well-designed IKS does more than merely present its stored knowledge to a user, it will: (1). Store information and present it quickly and efficiently. (2). Filter that information down to the essentials. (3). Help the user make a good decision. The rich knowledge-base of the Space Shuttle Hazardous Gas Detection System included in the IHM will provide HGDS personnel with a useful tool.

A functional IHM will be installed at HOSC, where its operational capabilities will be tested. The feedback from the NASA personnel will provide ideas for further improvement and expansion. Utilities which automatically incorporate new data will be added into the system to simplify the maintenance procedure. 


\section{Acknowledgements}

This work was supported under NASA Grant NAG8-835. The authors would like to thank R. D. Bachtel, H. Campbell, G. Lyles, and J. R. Redus of NASAMSFC for identifying the present topic and providing technical advice. Rockwell Inc. personnel are also appreciated for their technical support.

\section{References}

1. Conklin, J., "Hypertext: An Introduction and Survey," IEEE Computer, September, 1987.

2. Special Issue - "Hypertext" - communications of the ACM, July 1988.

3. Special Issue - "Hypertext-In-Depth Review", BYTE, October 1988.

4. Microsoft Windows 3.1, Microsoft Corp., 1992.

5. ToolBook 1.5, Assymetrix Corporation, 1989.

6. Guide 3 Professional Hypertext System, Owl International, Inc., 1990.

7. Origin 1.2. MicroCal, Inc., 1992.

8. DVA-4000 Full Motion Digital Video Adapter and Mic System 3.0 software, VideoLogic Limited, 1988. 


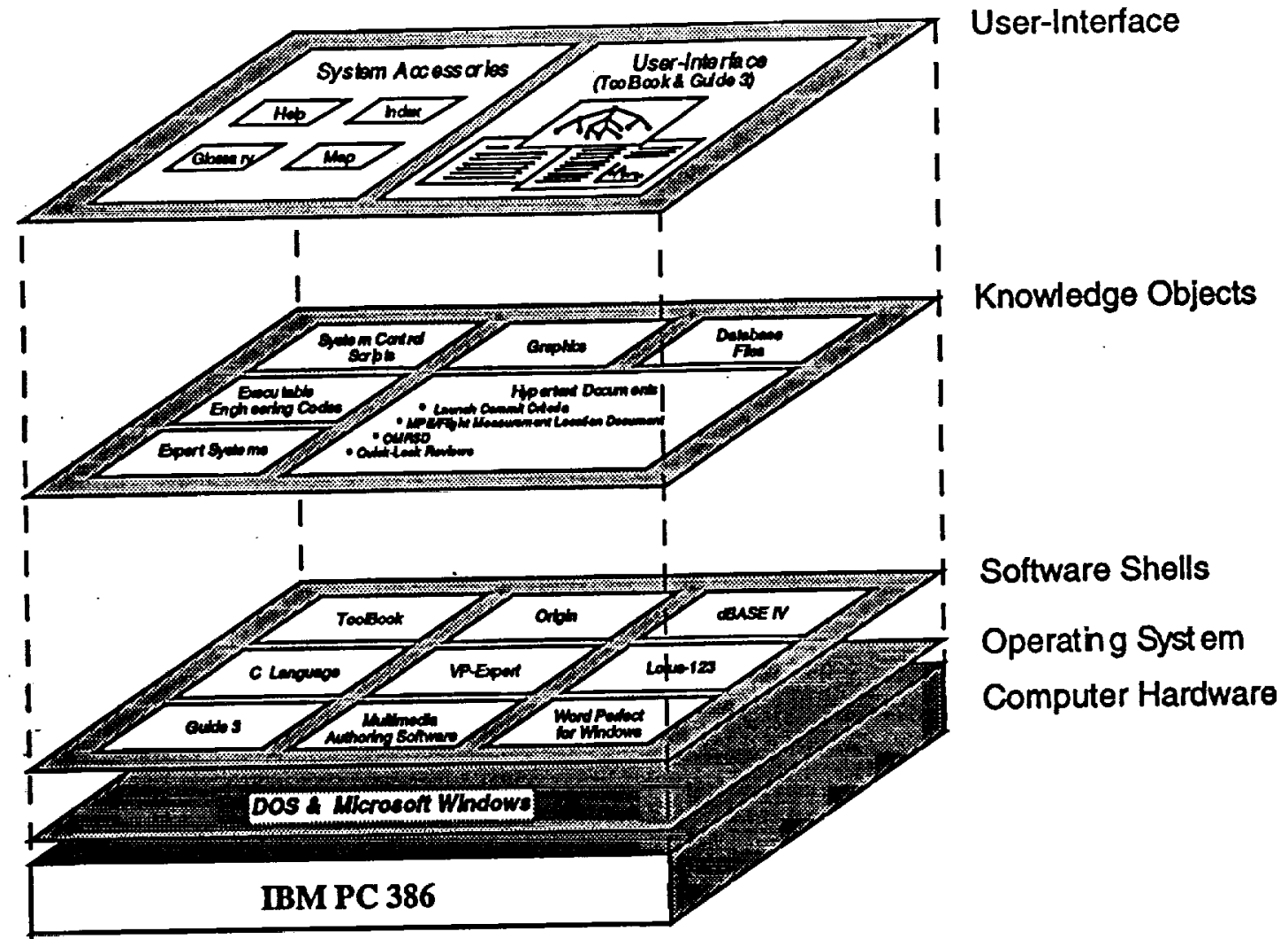

Figure 1. System architecture of the Intelligent Hypertext Manual.

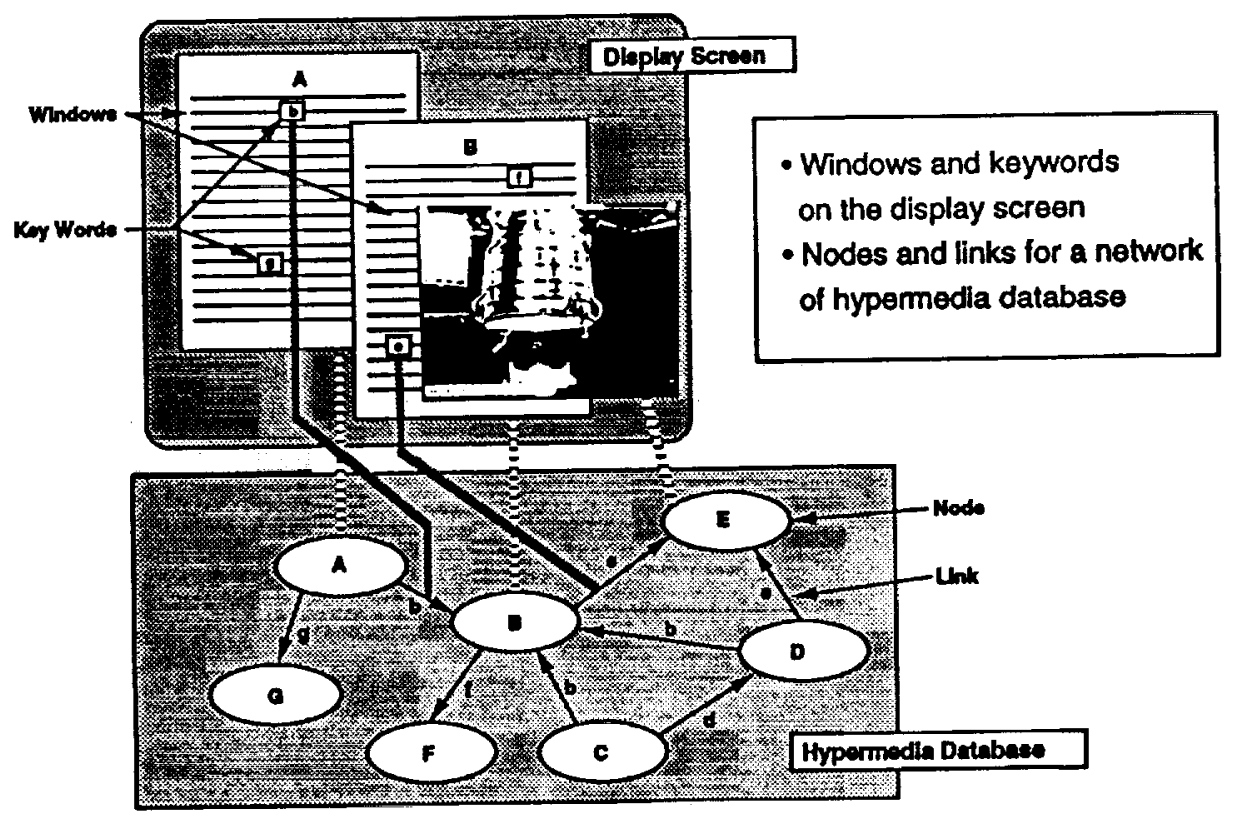

Figure 2. An illustration of hypermedia. 


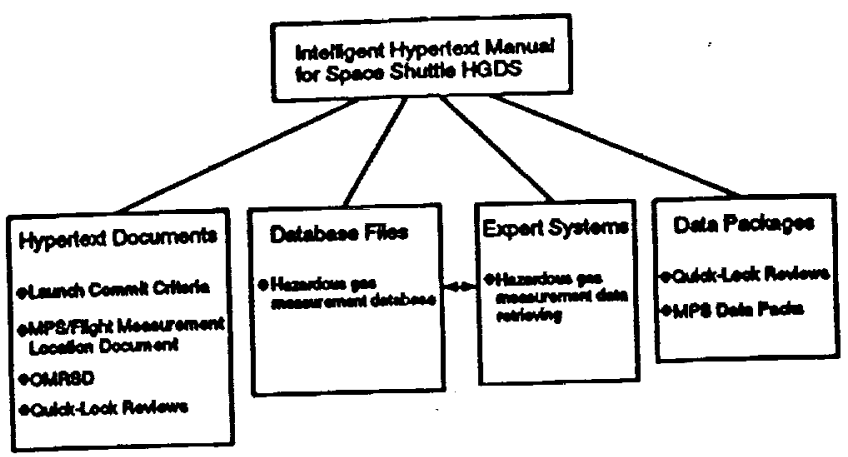

Figure 3. The knowledge-base of the Intelligent Hypertext Manual (IHM).

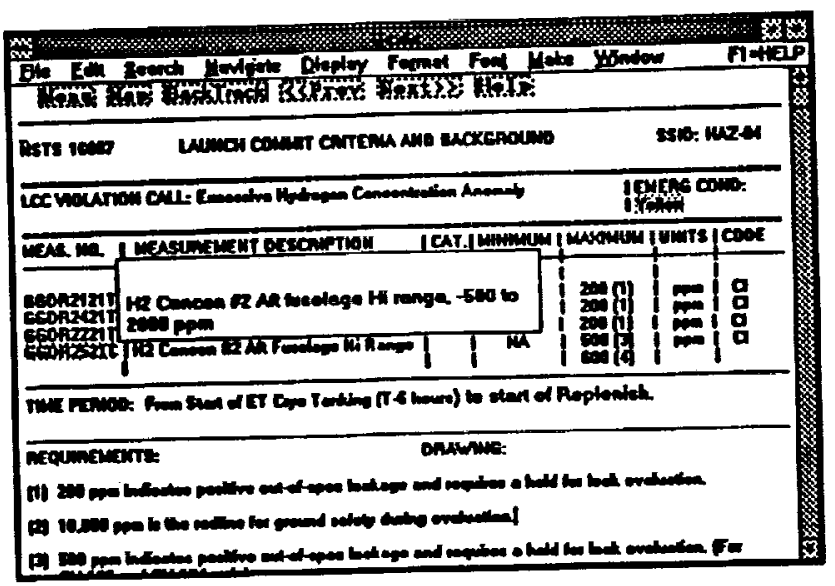

Figure 4. A screen display of LCC.

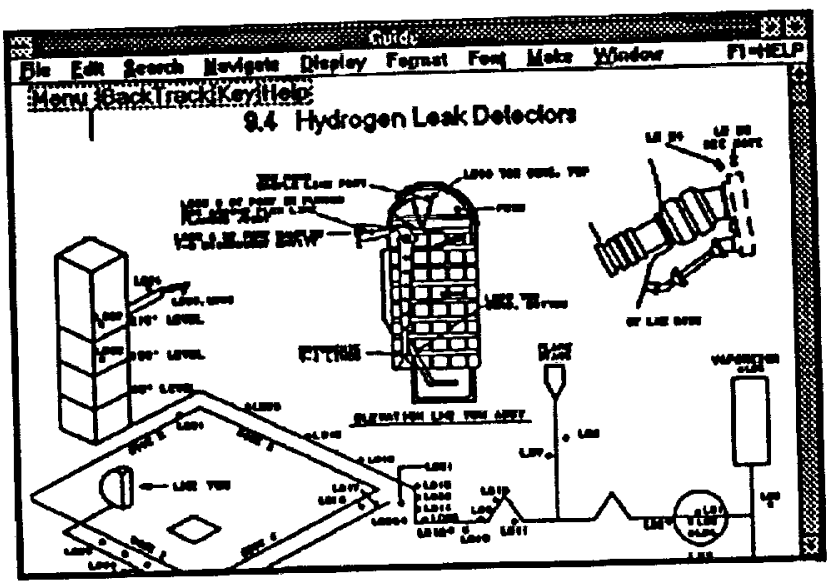

Figure 5. Display of a MPS/MLD document (Location of Hydrogen Leak Detectors).

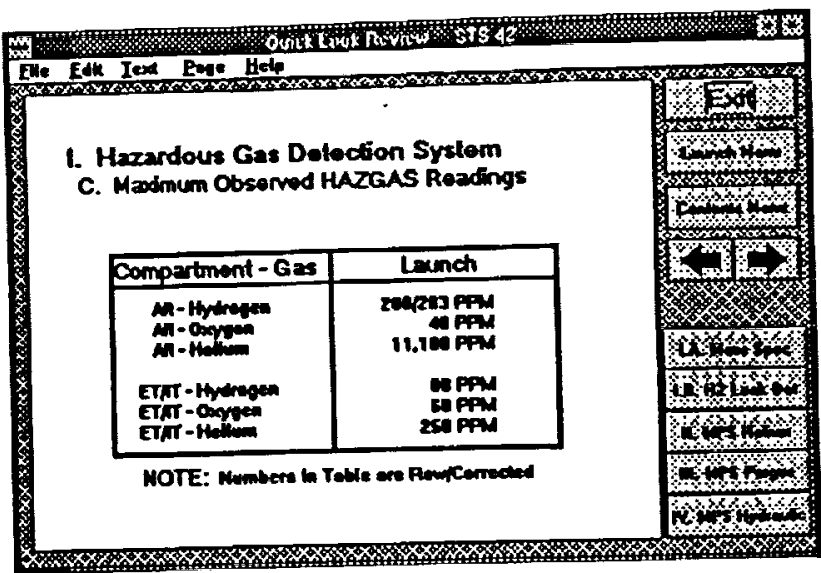

Figure 6. An example preface from the hypertext Quick-Look Reviews.

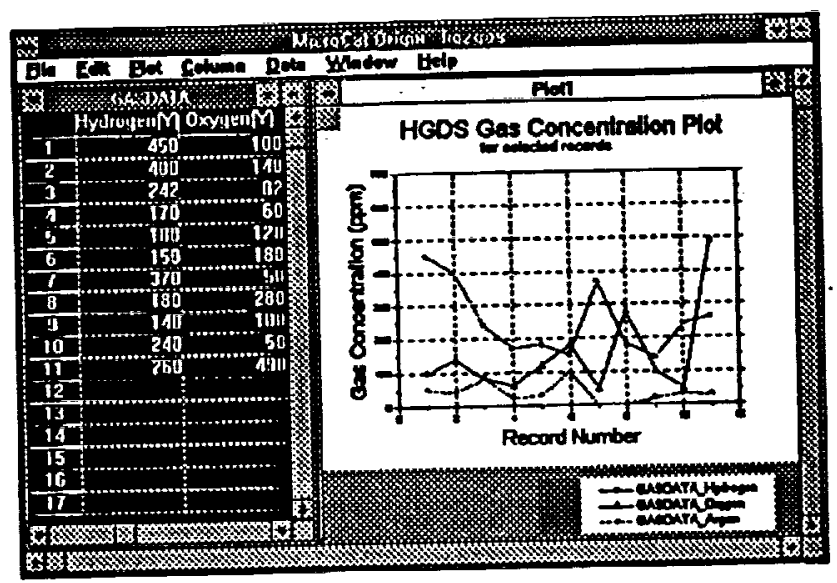

Figure 7. A Plot of sorted data from an expert system session.

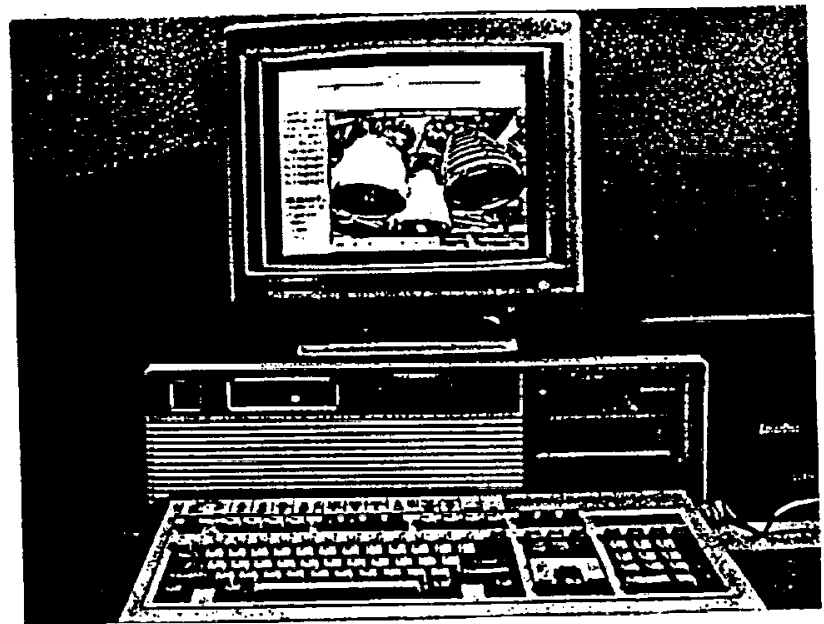

Figure 8. A sample hypermedia screen display. 